\title{
Towards Intra-operative 3D Nuclear Imaging: Reconstruction of 3D Radioactive Distributions Using Tracked Gamma Probes
}

\author{
Thomas Wendler ${ }^{1}$, Alexander Hartl ${ }^{1}$, Tobias Lasser ${ }^{1}$, Joerg Traub ${ }^{1}$, \\ Farhad Daghighian ${ }^{2}$, Sibylle I. Ziegler ${ }^{3}$, and Nassir Navab ${ }^{1}$ \\ ${ }^{1}$ Computer Aided Medical Procedures (CAMP), TUM, Munich, Germany \\ ${ }^{2}$ IntraMedical Imaging LLC, Los Angeles, California, USA \\ ${ }^{3}$ Nuclear Medicine Department, Klinikum rechts der Isar, TUM, Munich, Germany
}

\begin{abstract}
Nuclear medicine imaging modalities assist commonly in surgical guidance given their functional nature. However, when used in the operating room they present limitations. Pre-operative tomographic 3D imaging can only serve as a vague guidance intra-operatively, due to movement, deformation and changes in anatomy since the time of imaging, while standard intra-operative nuclear measurements are limited to 1D or (in some cases) 2D images with no depth information. To resolve this problem we propose the synchronized acquisition of position, orientation and readings of gamma probes intra-operatively to reconstruct a $3 \mathrm{D}$ activity volume. In contrast to conventional emission tomography, here, in a first proof-of-concept, the reconstruction succeeds without requiring symmetry in the positions and angles of acquisition, which allows greater flexibility. We present our results in phantom experiments for sentinel node lymph node localization. The results indicate that $3 \mathrm{D}$ intra-operative nuclear images can be generated in such a setup up to an accuracy equivalent to conventional SPECT systems. This technology has the potential to advance standard procedures towards intra-operative 3D nuclear imaging and offers a novel approach for robust and precise localization of functional information to facilitate less invasive, image-guided surgery.
\end{abstract}

\section{Introduction}

Nuclear medicine has become one of the most dynamic branches of medicine in today's diagnostic field. It is based on the use of radio-labeled, highly specific tracers that target functions in the body and can be imaged using gamma cameras, SPECT (single photon emission tomography) or PET (positron emission tomography) [1,2. In the particular case of SPECT a 3D gamma-emitting volume is reconstructed from several radial $2 \mathrm{D}$ orthographic projections acquired with gamma cameras 2. As in CT (computed tomography), the 3D volume reconstruction requires a complete set of projections and cylindrical symmetry of them. This and further issues like bulky equipment $\left(\approx 2 \times 2 \times 3\left[\mathrm{~m}^{3}\right]\right)$, resolution $(\approx 5[\mathrm{~mm}])$ and acquisition time $(\approx 20[\mathrm{~min}]$ pro bed position) make 3D nuclear imaging systems unsuitable for intra-operative use, so they are mostly restricted to diagnosis and planning. 
In order to overcome these limitations, hand-held non-imaging radioactivity detectors like gamma probes were introduced [3]. These are common diagnostic devices nowadays, especially during surgery and sentinel lymph node determination [4. The main advantages of these devices lie in their portability $(\approx 200[\mathrm{~g}]$, $\left.\approx 1 \times 1 \times 10\left[\mathrm{~cm}^{3}\right]\right)$, simplicity, and the possibility of miniaturizing them for intra-operative use. Hand-held gamma cameras have also entered the field recently [5], however the restrictions imposed by size $\left(\approx 10 \times 10 \times 50\left[\mathrm{~cm}^{3}\right]\right)$ and weight $(\approx 3[\mathrm{~kg}])$ limit their usefulness.

The combination of intra-operative nuclear devices with position and orientation ('pose') tracking has extended the use of this technology further. Wendler et al. introduced tracking of beta-probes for activity surface reconstruction, visualization and intra-operative guidance [6]. The beta radiation considered there was emitted from superficial tissue, and consequently, only an activity surface reconstruction was proposed. Benlloch et al. proposed tracking of $2 \mathrm{D}$ gamma cameras and the use of 2D acquisitions for limited 3D intra-operative functional imaging [7]. In that approach the $3 \mathrm{D}$ position is reconstructed from two $2 \mathrm{D}$ images taken with an angle close to 90 degrees or three $2 \mathrm{D}$ images with relative angles close to 120 degrees. However, these constraints greatly reduce the flexibility of this approach due the size of the devices and the requirements on the acquisition angles. Moreover, the detectable information is limited to point-like sources as a Computer Vision approach based on triangulation of corresponding points is taken for $3 \mathrm{D}$ reconstruction.

In this work we introduce a novel approach employing tracked gamma probes and algorithms for 3D tomographic reconstruction based on gamma readings and the synchronized 3D pose of the detector. Thus, most of the limitations complicating intra-operative nuclear imaging are removed. This approach includes the advantages of previous systems and surpasses them by allowing easier handling, faster acquisition times and most importantly, 3D intra-operative functional imaging of general distributions.

Applications like partial lymphadenectomy and sentinel lymph node detection [8,9] will greatly profit from the additional flexibility and the 3D nature of this kind of imaging. The added depth information allows detection of occlusions, aiding the resection of lymph nodes around a tumor, and increases the possibility to identify additional marked nodes not visible in pre-operative images, which are difficult to distinguish using conventional gamma probes or cameras (which additionally suffer from accessibility issues). Thus the proposed imaging modality will permit better intra-operative control of the operation yielding less invasive and more reliable procedures.

\section{Materials and Methods}

\subsection{Hardware Components}

Image acquisition is facilitated by a setup of a standard, collimated gamma probe together with an optical tracking system as outlined in figure 1, a detailed description can be found in 10 . 


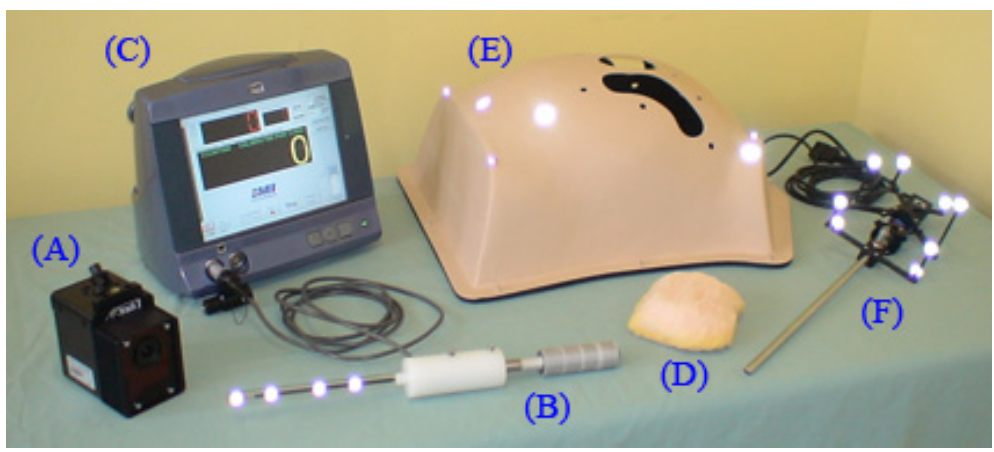

Fig. 1. The system setup consists of a 4-camera optical tracking system (exemplary camera marked as A), a gamma probe with a custom-made collimator and infrared markers to facilitate tracking (B) attached to a control unit (C), sending the data to a central workstation which also gathers the tracking data. A foam phantom with injected radioactivity (D) was placed inside the abdomen phantom (E), again with attached infrared markers. A tracked laparoscope (F) is used to generate an augmented reality visualization.

For validation, PET/CT images of the phantoms were generated a Biograph 16 PET/CT scanner by Siemens Medical Solutions (Erlangen, Germany).

Intra-operative 3D visualization as well as data synchronization was implemented by extending a framework for medical augmented reality systems [1]. A tracked and calibrated camera (Telecam SL NTSC by Karl Storz, Tuttlingen, Germany) was also added to the setup for augmented reality visualization. The calibration of this camera was done using the procedure described in 12 .

The visualization itself includes the rendering of the pre-operative PET/CT onto the registered image of the camera using 3D textures, where each voxel is rendered with a color and transparency as a function of its value. The most adequate choice of visualization was not part of the scope of this work, however it is subject of evaluation within our group [13.

During the scan, the positions of the acquired measurements are also augmented onto the scene as a point cloud (figure 2(a), color-coded according to the measured activity (no activity in green, red for positions with activity). For better 3D perception the points can optionally be displayed as vectors, adding the missing orientation component to the visualization. Afterwards, a volume rendering of the reconstructed volume is overlaid onto the image (figure 2(b)] . The user can acquire more points at any point if the reconstructed image is not of the desired quality. The different reconstruction methods may be switched on-the-fly.

\subsection{Modelling and Reconstruction}

The reconstruction problem in emission tomography consists of determining the activity distribution in a finite volume in space based on the readings of a sensor 


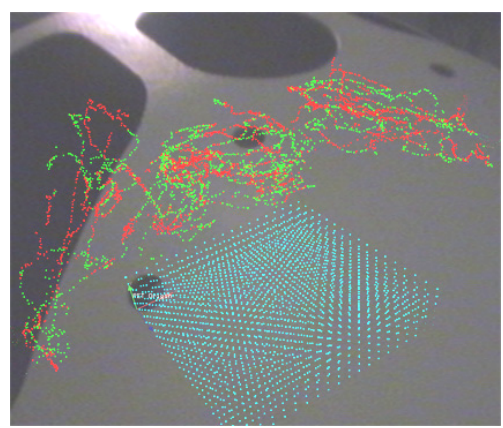

(a) AR view of measurements.

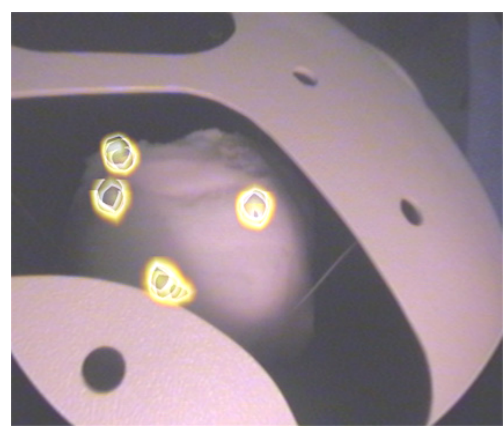

(b) AR view of reconstruction.

Fig. 2. (a) Green and red points mark the acquisition path of the gamma probe (red for active, green for inactive), the reconstruction grid is marked in cyan. (b) Reconstructed activity distribution marked in yellow.

array, for example a SPECT device. Let $\boldsymbol{x}_{j}, j=1, \ldots, M$, denote an equidistantly spaced discrete $3 \mathrm{D}$ grid of voxels, where each voxel $\boldsymbol{x}_{j}$ has a total activity of $f_{j}$. Further let $g_{i}$ denote the reading of each sensor $i$ in the array, $i=1, \ldots, N$. As in standard practice, we assume that each reading $g_{i}$ is a linear function of the activity $f_{j}$ of all voxels $\boldsymbol{x}_{j}$, in short

$$
g_{i}=\sum_{j=1}^{M} H_{i j} f_{j} .
$$

For a given sensor $i, H_{i j}$ denotes a vector correlating the effect of the acitivities $f_{j}$ at positions $\boldsymbol{x}_{j}, j=1, \ldots, M$, to the sensor reading $g_{i}$. The entire matrix $H_{i j}$ is also known as the system matrix, or as the forward model describing radiation propagation through the volume to the detector positions. The process of reconstruction then solves the ill-posed problem of determining the set $\left(f_{j}\right)_{j=1, \ldots, M}$ given the readings of the $N$ sensors, or in terms of equations, the solution of the linear equation system formed by equation (11) for $i=1, \ldots, N$.

In the case of intra-operative gamma probes, each probe readout $g_{i}$ is to be accompanied by a position and orientation vector pair $\left(\boldsymbol{p}_{i}, \hat{d}_{i}\right)$ provided by the tracking system. These readings can be considered independently, assigning to each of them an own row in the system matrix. $H_{i j}$ represents in this case the influence of the activity in voxel $j$ on the readout of the probe at the pose $\left(\boldsymbol{p}_{i}, \hat{d}_{i}\right)$. Care has to be taken to ensure a synchronized readout and that the coordinate systems of the tracked probe and the reconstruction grid are properly co-calibrated.

To determine the system matrix $\left(H_{i j}\right)$ a forward model has to be developed, describing the propagation of gamma radiation through space and characterizing the detection process at the sensor. In our model we consider the field of view and the sensitivity of the probe, the stochastic nature of radioactive decay and detection as well as the absorption in the detector, geometrical attenuation and 
background noise. More details of this modelling process can be found in [14]. To determine the unknown variables a set of measurements was acquired at different distances and positions from the probe and the parameters are fitted to these measurements using a best-neighbor optimizer.

To solve the ill-posed equation system (1) many algorithms have been proposed for SPECT and PET 2. In our implementation we used both algebraic (randomized algebraic reconstruction technique, ART) and stochastic approaches (maximum-likelihood expectation maximization, ML-EM) to obtain an approximated solution. We also evaluated other numerical tools such as SVD with Tikhonov regularization [15] to approximately solve the linear system.

\section{Experiments}

To validate the developed theory a set of phantom experiments was performed. A foam phantom of an organ was injected in 4 positions with a double marked solution containing $T c-99 m$ and $F-18(50[k B q]$ and $20[k B q]$ respectively per milliliter) to simulate marked lymph nodes as one expect in a sentinel lymph node localization procedure. F-18 was used to enable imaging with $\mathrm{PET} / \mathrm{CT}$ and did not influence the gamma probe readings (sensitivity of $5.6[\mathrm{cps} / \mathrm{MBq}$ ] for a point source of $F-18$ at a distance of $5[\mathrm{~cm}]$ along the axis of the probe).

After acquiring a PET/CT (5 $[\mathrm{min}]$ per bed position) of the phantom, several gamma probe scans were performed for each experiment by three different test persons. Slight movement and deformation of the phantom during the scan was permitted to introduce realistic artifacts. The test persons were able to do reconstructions on-line to assess the quality of the generated images and plan further acquisition paths. The points already acquired were also augmented onto the image for this reason. The voxel size used was $5.3 \times 5.3 \times 6\left[\mathrm{~mm}^{3}\right]$ (similar to the one of a conventional SPECT) for a volume of $20 \times 7 \times 17$ (width $\times$ height $\times$ depth). The number of iterations for ML-EM and ART were fixed empirically to yield qualitative good results (14 and 20 iterations respectively) and the initial guess was set to 0 .

\section{Results}

The experiments yielded promising results. The qualitative comparison of the reconstructed images with PET is satisfying up to the level that it may be considered for intra-operative image-guidance (figures 3(a) vs. 3(b)] .

The quantitative comparison using normalized cross-correlation and the deviation of the centroid of the detected blobs from the ones visible in PET is outlined in Table (11). These measurements include not only the reconstruction error (when solving the linear system) but also the registration error of the probe and the reconstruction grid as well as tracking inaccuracy, and as such may be used as system performance measure.

The variant of randomized ART employed is not performing well as an inversion routine for our forward model, both quantitatively and qualitatively, as seen 


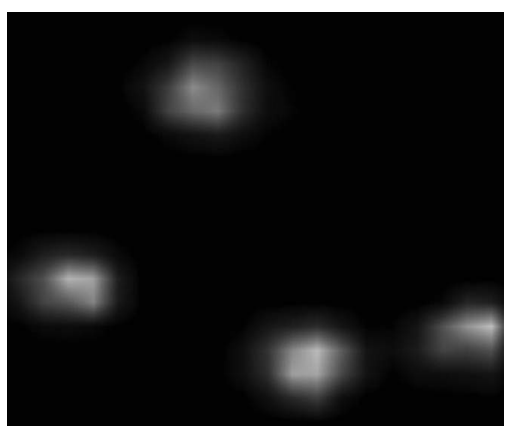

(a) Reconstructed slice.

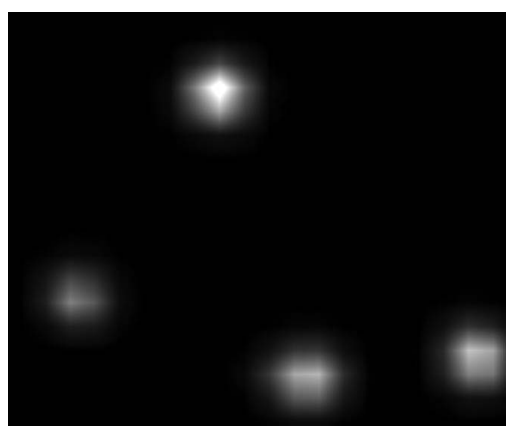

(b) Corresponding PET slice.

Fig. 3. Comparison of one reconstructed slice and the corresponding PET slice. MLEM was used for inversion, the acquisition consisted of 5198 readings $(\approx 6[\mathrm{~min}])$.

Table 1. Evaluation of quality of the reconstructed images versus PET for different inversion schemes. The first column contains the normalized cross-correlation of both modalities, the remaining columns display the mean deviation in $[\mathrm{mm}]$ of the centroids of the detected objects in the reconstruction from the ones visible in PET. Values are given as mean \pm standard deviation for the experiments.

\begin{tabular}{c||c|c|c|c} 
Method & NCC & Deviation in $x$ & Deviation in $y$ & Deviation in $z$ \\
\hline \hline ART & $0.252 \pm 0.199$ & $2.686 \pm 9.020$ & $3.488 \pm 8.836$ & $1.251 \pm 5.570$ \\
ML-EM & $0.512 \pm 0.077$ & $0.778 \pm 2.973$ & $3.502 \pm 3.674$ & $2.717 \pm 4.174$ \\
SVD & $0.521 \pm 0.014$ & $0.167 \pm 4.775$ & $2.017 \pm 3.798$ & $6.136 \pm 4.156$
\end{tabular}

in Table (1). Reconstructions using regularized SVD inversion yield good qualitative results, while being visually adequate. Our clear method of choice is ML-EM. This algorithm is fast (less than 20 seconds in a up-to-date standard PC) and the reconstruction evince good quantitative and qualitative performance.

Deviations in the $y$ axis (dorsal direction) are more marked than in the other dimensions. This is due to the nature of the acquisition where readings are mainly acquired on top of the abdomen phantom and thus lacking projections to better resolve the dorsal axis. Overall however, deviations are on the order of the pixel size of the reconstruction grid.

\section{Discussion}

Gamma probes have been used for sentinel lypmh node determination for years [3, 4. The high sensitivity and specificity values achieved speak of a robust technique especially for melanoma and breast cancer [8, 9]. Hand-held gamma cameras enhance this technique even further by adding imaging [5]. The inclusion of 3D imaging thus would not improve the standard technique significantly in terms of sensitivity and specificity. However, we believe that the major 
contribution of this work is the gain of full 3D perception of the node distribution and thus the capability of allowing precise localization in depth, which would be almost impossible with the current technology. This is especially true in the case of a partial lymphadenectomy where current technology only allows a rough distinction of the affected nodes resulting in suboptimal resection and higher morbidity.

The presence of background activity is an issue to be investigated in the future mostly for molecular markers like F-18-FDG. Here most probably the sparse information would not be enough to guarantee a valid reconstruction that is comparable to pre-operative imaging systems. A solution for this could be the use of compressed sensing approaches like the ones proposed in [16.

In the case of the proposed applications (partial lymphadenectomy and sentinel lymph node localization), this however does not play any role, since the marking is achieved by injecting radioactivity before resection, thus making valid the assumption that at a certain instant in time only downstream lymph nodes will present radioactive uptake (several hot spots and almost no background).

The inclusion of tracking into the operation room should not change the workflow dramatically, nor add much complexity to the operation room. A relevant issue is the robustness of optical tracking systems mostly in terms of occlusion problems. We believe that this will not be a major issue in this application if several cameras are placed in the operation room. Furthermore, the scan can be performed by one person while the surgical team can remain at a proper distance from the patient, thus avoiding occlusions and guaranteeing a better tracking. The influence of the proposed changes in the work-flow is subject of current research.

In regard to the patient dose, our system would require no extra activity than the one used for radio-guided lymphadenectomy or sentinel lymph node localization $(\approx 2[m S v]$ and $<1[m S v]$ respectively versus the $5-7[\mathrm{mSv}]$ of pre-operative imaging). We believe that this burden can be neglected when considering the improvement in therapy we aim at.

The reconstructed images are valid as long as the reconstructed region containing the activity does not move or deform. The intra-operative, on-the-fly nature of the reconstruction makes it valuable for correction and deformation of the pre-operative imaging data and thus update the surgical plan. This will enable more precise intra-operative localization. The imaging process is short and can be repeated as many times as needed.

A final issue is the fact that the reconstruction obtained shows a blurring of the activity blobs in dorsal direction. This effect can be explained due to the missing information in that axis for the reconstruction. However, since the blobs can clearly be recognized in the images and their upper border is placed correctly when compared with the PET images, we strongly believe that the images are sufficient for precise localization in the suggested applications, which was previously not possible at all. Overall accuracy of the reconstructions are within theoretical system limits and are on par with conventional SPECT systems 
in terms of resolution and contrast. Defining if the current implementation would make sense for specific clinical applications in terms of specifications is part of our work-in-progress.

\section{Conclusions}

This paper presents an approach towards intra-operative 3D nuclear imaging employing a tracked gamma probe. The resulting reconstruction accuracy is comparable to SPECT and suggests further development and clinical evaluation of this technique. In addition, the tracking system can further be taken advantage of by using it to track surgical instruments enabling navigation within the same coordinate system as the reconstruction. For the proposed applications in particular this would enable guided biopsy of the sentinel lymph nodes and precise node resection in partial lymphadenectomy.

\section{References}

1. Phelps, M.E.: PET: The merging of biology and imaging into molecular imaging. J. Nuc. Med. 41, 661-681 (2000)

2. Wernick, M.N., Aarsvold, J.N.: Emission Tomography: The Fundamentals of PET and SPECT. Academic Press, London (2004)

3. Hoffman, E.J., et al.: Intraoperative probes and imaging probes. Eur. J. Nucl. Med. Mol. Imaging 26, 913-935 (1999)

4. Harish, K.: Sentinel node biopsy: concepts and current status. Front. Biosci. 10, 2618-2644 (2005)

5. Pitre, S., et al.: A hand-held imaging probe for radio-guided surgery: physical performance and preliminary clinical experience. Eur. J. Nucl. Med. Mol. Imaging 30, 339-343 (2003)

6. Wendler, T., et al.: Navigated three dimensional beta probe for optimal cancer resection. In: Larsen, R., Nielsen, M., Sporring, J. (eds.) MICCAI 2006. LNCS, vol. 4190, pp. 561-569. Springer, Heidelberg (2006)

7. Benlloch, J.M., et al.: The gamma functional navigator. IEEE Trans. Nucl. Sci. 51, 682-689 (2004)

8. Focht, S.L.: Lymphatic mapping and sentinel lymph node biopsy. AORN J 69, 802-809 (1999)

9. Reintgen, D., et al.: Lymphatic mapping and sentinel lymph node biopsy for breast cancer. Cancer J. 8 (Suppl. 1), 15-21 (2002)

10. Wendler, T., et al.: Real-time fusion of ultrasound and gamma probe for navigated localization of liver metastases. In: Larsen, R., Nielsen, M., Sporring, J. (eds.) MICCAI 2006. LNCS, vol. 4190, Springer, Heidelberg (2006)

11. Sielhorst, T., et al.: Campar: A software framework guaranteeing quality for medical augmented reality. International Journal of Computer Assisted Radiology and Surgery 1(Supp. 1), 29-30 (2006)

12. Feuerstein, M., et al.: Automatic patient registration for port placement in minimally invasive endoscopic surgery. In: Larsen, R., Nielsen, M., Sporring, J. (eds.) MICCAI 2006. LNCS, vol. 4190, pp. 287-294. Springer, Heidelberg (2006) 
13. Traub, J., et al.: Hybrid navigation interface for orthopedic and trauma surgery. In: Larsen, R., Nielsen, M., Sporring, J. (eds.) MICCAI 2006. LNCS, vol. 4190, pp. 373-380. Springer, Heidelberg (2006)

14. Hartl, A.: Gamma-probe modeling for reconstruction. Technical report, Computer Aided Medical Procedures (CAMP), TUM, Munich, Germany (2007)

15. Hansen, P.C.: Rank-Deficient and Discrete Ill-Posed Problems: Numerical Aspects of Linear Inversion. SIAM, Philadelphia (1998)

16. Wakin, M., et al.: An architecture for compressive imaging. In: Proc. ICIP 2006, Atlanta, GA (2006) 